\title{
ASSOCIAÇÃO DE EVENTOS ESTRESSORES E MORBIDADE PSIQUIÁTRICA EM GESTANTES*
}

\section{Larissa Horta Esper ${ }^{1}$; Erikson Felipe Furtado ${ }^{2}$}

A literatura aponta importantes associações entre a vivência de eventos estressores por gestantes e prejuízos à saúde mental materna. Este estudo teve por objetivo analisar a relação entre a ocorrência de eventos estressores e a morbidade psiquiátrica no terceiro trimestre da gestação. Trata-se de estudo epidemiológico clínico, observacional, transversal, sobre amostra de 449 gestantes, recrutadas de forma sequencial e aleatória em uma maternidade pública na cidade de Ribeirão Preto, SP. Os dados foram coletados através dos seguintes instrumentos: um questionário estruturado, visando a obtenção de dados sociodemográficos, histórico gestacional e saúde da gestante, um questionário de morbidade psiquiátrica (QMPA) e uma entrevista semiestruturada para eventos recentes de vida (IRLE). Todas as participantes apresentaram eventos estressores $(\mathrm{m}=5 ; \mathrm{dp}=2,2 ; \min =1$-máx $=14)$ sendo a categoria saúde a mais frequente $(99,1 \%)$ e os eventos "gravidez indesejada" $(60,58 \%$; $=272)$ e "dificuldade financeira média" $(31,85 \% ; n=143)$ os mais comuns. O número total de eventos estressores apresentou correlação positiva e significante em relação aos sintomas e queixas emocionais de gestantes $(\mathrm{p}<0,01)$. Quando avaliados separadamente por categorias, "finanças" foi a categoria mais fortemente relacionado $(p<0,01)$. Os resultados apontam alta vivência materna de eventos estressores durante o período gestacional e correlação positiva entre eventos estressores e sintomas psiquiátricos.

Descritores: Gravidez; Estresse Psicológico; Sintomas Afetivos.

\section{THE ASSOCIATION OF STRESSFUL EVENTS AND PSYCHIATRIC MORBIDITY IN PREGNANT WOMEN $^{\dagger}$}

The scientific literature points out the occurrence of relevant associations between stressful experiences and mental health problems among pregnant women. The objective of this study was to analyze the relationship between stressful events and psychiatric morbidity in the third trimester of pregnancy. The study has a clinical, epidemiological, observational, crosssectional design performed on a sample of 449 pregnant women recruited in a sequential and random fashion at a public maternity hospital in Ribeirão Preto (São Paulo). Data collection was performed using the following instruments: one structured questionnaire, aiming at obtaining socio-demographic data, clinical history of the pregnancy and of the woman's health, a questionnaire on psychiatric morbidity (QMPA) and a semi-structured interview for recent life events (IRLE). All participants had experienced stressful events $(\mathrm{m}=5 ; \mathrm{sd}=2.2$; $\min =1-\max =14)$ with the health category being the most frequent $(99.1 \%)$ and the most common events being "unwanted pregnancy" $(60.58 \% ; n=272)$ and "average financial burden" $(31.85 \% ; n=143)$. The total number of stressful events had a positive and significant

\footnotetext{
* Trabalho apresentado no XI Encontro de Pesquisadores em Saúde Mental e Enfermagem Psiquiátrica. Ribeirão Preto, SP, Brasil, 2010.

${ }^{\dagger}$ This study was presented in the $11^{\text {st }}$ Meeting of Researchers in Mental Health and Psychiatric Nursing. Ribeirão Preto, SP, Brazil, 2010.
}

1-Enfermeira, Mestranda em Saúde Mental, Faculdade de Medicina de Ribeirão Preto, Universidade de São Paulo, SP, Brasil. E-mail larissa.esper@usp.br

2-Psiquiatra, Doutor em Medicina, Professor Doutor, Faculdade de Medicina de Ribeirão Preto, Universidade de São Paulo, SP, Brasil. E-mail efurtado@,fmrp.usp.br

Autor correspondente: Larissa Horta Esper Endereço para Correspondência: Universidade de São Paulo, Faculdade de Medicina de Ribeirão Preto, Hospital das Clínicas, $3^{\circ}$ Andar, Sala 333 Avenida Bandeirantes, 3900, Monte Alegre, CEP 14049-400, Ribeirão Preto, SP, Brasil.E-mail: larissa.esper@usp.br 
correlation to symptoms and emotional complaints of the women $(p<0.01)$. When the categories were evaluated separately, "finances" had the strongest relationship $(p<0.01)$. Results show that women experience many stressful events during pregnancy, and that there is a positive correlation between stressful events and psychiatric symptoms.

Descriptors: Pregnancy; Stress, Psychological; Affective Symptoms.

ASOCIACIÓN DE EVENTOS ESTRESORES Y MORBILIDAD PSIQUIÁTRICA EN GESTANTES

La literatura reseña importantes asociaciones entre la vivencia de eventos estresores por parte de gestantes y perjuicios a la salud mental materna. Este estudio tuvo como objetivo analizar la relación entre la ocurrencia de eventos estresores y la morbilidad psiquiátrica en el tercer trimestre de la gestación. Se trata de un estudio epidemiológico clínico, observacional, transversal, sobre muestra de 449 gestantes reclutadas en forma secuencial y aleatoria en una maternidad pública en la ciudad de Ribeirão Preto-SP. Los datos fueron recolectados a través de los siguientes instrumentos: un cuestionario estructurado, apuntando a la obtención de datos sociodemográficos, histórico gestacional y salud de la gestante, un cuestionario de morbilidad psiquiátrica (QMPA) y una entrevista semiestructurada para eventos recientes de vida (IRLE). Todas las participantes presentaron eventos estresores $(\mathrm{m}=5 ; \mathrm{dp}=2,2 ; \mathrm{min}=1-$ máx $=14)$ resultando la categoría salud la más frecuente $(99,1 \%)$ y los eventos "embarazo no deseado" (60,58\%; $n=272)$ y "dificultad financiera media" $(31,58 \% ; n=143)$ los más comunes. El número total de eventos estresores presentó una correlación positiva y significativa en relación a los síntomas y quejas emocionales de las gestantes $(p<0,01)$. Cuando fueron evaluados en forma separada por categorías, "finanzas" fue la más fuertemente relacionada $(\mathrm{p}<0,01)$. Los resultados demuestran alta vivencia materna de eventos estresores durante el período gestacional y una correlación positiva entre eventos estresores y síntomas psiquiátricos.

Descriptores: Embarazo, Estrés Psicológico, Síntomas Afectivos.

${ }^{\ddagger}$ Trabajo presentado en el XI Encuentro de Investigadores en Salud Mental y Enfermería Psiquiátrica. Ribeirão Preto, SP, Brasil, 2010. 


\section{Introdução}

O período gestacional é importante momento de mudanças físicas, sociais, familiares e psicológicas, no qual a mulher fica exposta a diversas situações estressantes, podendo gerar prejuízos à sua saúde mental. Os estudos acerca do papel das variáveis psicossociais, como fatores de risco para o desencadeamento de doenças psiquiátricas, têm demonstrado a importância de identificar as variáveis ambientais que influenciam no fenômeno da doença mental, sendo o reconhecimento dos eventos estressores de extrema relevância para esclarecer o desencadeamento de diversas doenças, durante a gestação.

Os eventos estressores se referem a ocorrências externas, identificadas no tempo, que podem gerar mudanças pessoais e sociais no ambiente do sujeito $^{(1-3)}$, geralmente são causadores de estresse devido à necessidade de novas adaptações do indivíduo ao meio. São exemplos de ocorrências externas situações como mudança de emprego, separação conjugal, morte de familiares, dificuldades financeiras entre outros. As doenças físicas e a gravidez, apesar de serem ocorrências internas, são importantes eventos estressores considerados, pois podem exigir do indivíduo alterações no seu ambiente social e pessoal, assim como ocorre nos acontecimentos meramente externos.

A literatura tem demonstrado que o acúmulo de eventos estressores pode gerar importantes danos à saúde física e mental da gestante. Em recente estudo, foi encontrado que gestantes depressivas, quando comparadas a um grupo controle, reportam mais eventos estressores, percepção de impacto negativo mais 
intenso e maior prevalência de eventos independentes (eventos que possivelmente não são consequência da doença psiquiátrica) $)^{(4)}$.

Fatores relacionados ao estresse estão associados a diversos prejuízos à saúde da mãe durante o período gestacional, parto e puerpério. Os altos níveis de estresse e ansiedade, durante a gestação, parecem aumentar os riscos para aborto espontâneo, trabalho de parto prematuro e complicações obstétricas ${ }^{(5-7)}$.

Em estudo recente ${ }^{(5)}$, realizado com mães americanas, foram encontradas importantes associações entre vivência de eventos estressores e a maior ocorrência de partos prematuros. Dentre os 18 eventos avaliados, a autora destaca os problemas financeiros, a violência doméstica, o divórcio e a ocorrência de atentados de suicídio por familiares ou amigos próximos da gestante como os principais estressores que coincidem com o aumento de risco para parto prematuro.

A vivência de eventos estressores severos, tais como violência do companheiro, parece estar associada também ao aumento de consumo de substâncias como o álcool, durante o período gestacional $^{(5,7)}$. Outros estudos encontraram ainda associações entre os transtornos relacionados ao consumo de álcool, maior sofrimento psiquiátrico e sintomas depressivos e ansiosos entre gestantes $^{(8-9)}$.

O desenvolvimento do bebê também é prejudicado pelo estresse gestacional. A literatura demonstra que os elevados níveis de cortisol materno, liberados em resposta ao estresse físico ou psicológico, produzem permanentes alterações no eixo hipotalâmicopituitário-adrenal do feto, gerando importantes danos ao seu 
desenvolvimento. Tal modificação ansiedade e depressão que podem aumenta as probabilidades de aparecer nessas fases. O apoio emocional malformações congênitas, como fissuras do companheiro e o apoio familiar podem palatinas, em filhos de mulheres com gestações indesejadas e deformações cranioneurais $^{(10-12)}$.

Os efeitos do estresse materno em fases tardias da gestação parecem estar relacionados, ainda, a alterações de ordem emocional e comportamental de crianças $^{(11)}$. A ansiedade pode estar associada à hiperatividade e ao déficit de atenção em meninos e problemas emocionais e comportamentais em meninas, na idade de quatro anos.

A literatura aponta que o ambiente pré-natal conturbado da gestante parece contribuir para o baixo peso do bebê ao nascer $^{(12-13)}$ e verificam, ainda, que o adequado suporte social, proporcionado durante os períodos gestacional, parto e puerpério favorece a mulher ao diminuir a 


\section{Objetivos}

Analisar a relação entre a ocorrência de eventos estressores e a morbidade psiquiátrica de mulheres no terceiro trimestre da gestação. atende pelo SUS, no município de Ribeirão Preto, SP. Os critérios para inclusão no estudo foram: ausência de gestação de risco, estar em condições de oferecer informações e declarar seu consentimento informado.

\section{Coleta de dados}

\section{Materiais e métodos}

Tipo de estudo

Este é um estudo epidemiológico clínico, observacional, transversal, com dois momentos de levantamento de dados, sobre amostra de conveniência, recrutada de forma sequencial e aleatória.

Participantes da pesquisa

As participantes da pesquisa foram gestantes no terceiro trimestre de gestação, usuárias da maternidade que
A coleta de dados ocorreu na maternidade municipal. As entrevistas foram realizadas na ocasião das consultas de rotina para avaliação obstétrica das gestantes, durante o período de 2001 a 2002. Os entrevistadores foram duas psiquiatras e uma enfermeira em esquema de rodízio diário.

\section{Procedimentos}

A observação transversal foi constituída através da realização de uma 
única entrevista, sendo dividida em dois momentos. No primeiro, foi aplicado um questionário estruturado, visando, com esse, a obtenção de dados sociodemográficos, histórico gestacional, saúde da gestante e padrão de consumo de álcool. No segundo momento, a gestante respondeu questões de dois instrumentos: QMPA (Questionário de Morbidade Psiquiátrica) e IRLE (Interview for Recent Life Events). A amostra final ficou composta por 449 gestantes.

Instrumentos

Durante a coleta de dados foram utilizados os instrumentos, mostrados a seguir.

- Ficha de registros de dados, a qual contém informações referentes à identificação da paciente, dados sociodemográficos, dados do prontuário da paciente e dados colhidos pela gestante.

- QMPA (Questionário de Morbidades Psiquiátricas) foi desenvolvido e adaptado às especificidades regionais da linguagem $\quad$ brasileira $^{(16)}$, construído para ser autoaplicável ou aplicado por entrevistador leigo e respondido por terceiros. As alternativas de resposta são sim ou não, para as quais são atribuídos valores de um ou zero. As primeiras 43 questões se referem ao próprio indivíduo, do qual se quer saber o estado de saúde mental, e as duas últimas questões (44 e 45) referem-se à sintomatologia apresentada por qualquer um dos membros da família. Um ponto de corte igual ou superior a sete (7) denota 
suspeição de transtorno

psiquiátrico.

- IRLE (Interview for Recent Life Events), esse instrumento avalia os eventos estressores, o mês de ocorrência e o nível de estresse referido pelo entrevistado ${ }^{(1,17)}$.

Trata-se de entrevista

semiestruturada, composta por 64 itens agrupados em 10 categorias: trabalho, educação, finanças, saúde, reação de luto ou perda, migração, namoro e casamento, aspectos legais, família e sociedade e relações conjugais. Apenas um dos 64 eventos não é especificado pelo instrumento, permitindo a anotação de algum outro acontecimento que não tenha sido citado anteriormente. A entrevista não apresenta um sistema de pontuação para análise. O agrupamento de eventos tem sido empregado pelos pesquisadores para examinar os indivíduos com um ou mais eventos de vida. Na literatura científica internacional, há inúmeros exemplos de pesquisas realizadas por meio desse instrumento, a fim de estudar as relações entre os eventos estressores e diversos transtornos mentais ${ }^{(17-19)}$

\section{Análise estatística}

O programa SPSS versão 17.01 foi utilizado para realizar as análises estatísticas descritivas (frequências simples, porcentagens, médias e desvio padrão) e para caracterizar a amostra, segundo variáveis sociodemográficas definidas (idade, estado civil, renda familiar e religião). Essa mesma estratégia estatística foi adotada para apresentar os escores do instrumento QMPA e os dados do IRLE.

$\mathrm{Na}$ avaliação da diferença de médias entre os grupos, foi utilizado o 
teste não-paramétrico de Kruskal-Wallis.

O nível de significância adotado foi de $5 \%$ para todos os testes $(\mathrm{p} \leq 0,05)$.

Aspectos éticos

Este projeto é recorte e faz parte de um projeto principal intitulado GestaÁlcool-Estudo Longitudinal sobre consumo de álcool durante a gestação. O projeto de origem teve aprovação pelo Comitê de Ética em Pesquisa do Hospital das Clínicas da Faculdade de Medicina de Ribeirão Preto da Universidade de São Paulo.

\section{Resultados}

A amostra foi composta por 449 gestantes, dessas, a maioria jovem $(m=23,9 ; \quad d p=5,51), \quad$ branca $\quad(58,8 \%)$, casada (relacionamento consensual ou legal) $(85,3 \%)$ e tinham prática religiosa
$(57,7 \%)$. Quanto à escolaridade, $1,3 \%$ não tinha escolaridade, $63,4 \%$ das gestantes apresentavam escolaridade até o nível fundamental, 35,2\% apresentavam nível médio e apenas $0,9 \%$ tinham nível superior

A maioria tinha baixa renda per capita, sendo que $71,7 \%$ apresentavam renda de até cinco salários mínimos, 24,7 \% recebiam de cinco a dez salários mínimos e apenas 3,6\% tinham renda maior que dez salários mínimos.

Em relação ao número de gestações, aproximadamente 188 das gestantes $(41,9 \%)$ eram primigestas, comparadas com 260 (58\%) que eram multíparas, dessas $114 \quad(25,4 \%)$ apresentavam duas gestações prévias, 58 $(13 \%)$ três gestações, $45(10 \%)$ quatro gestações, 23 (5,1\%) com cinco gestações e apenas $21(4,6 \%)$ apresentavam mais de cinco gestações. 
Os eventos estressores foram Quando avaliados separadamente medidos através do instrumento IRLE. por categorias, os eventos relacionados à Trata-se de inventário composto por 64 saúde foram citados com maior itens, divididos em dez categorias que frequência (99,1\%), seguidos dos eventos verifica a ocorrência de diferentes eventos da categoria família e sociedade $(51,0 \%)$, estressores e a percepção de impacto de relações conjugais (48,6\%), finanças estresse, diante de tais vivências. Todas (42,1\%), trabalho (34,3\%), migração as gestantes do estudo relataram (32,7\%), reação de luto ou perda $(31,4 \%)$, ocorrência de pelo menos um evento educação $(25,4 \%)$, aspectos legais estressor durante os 12 meses anteriores à $\quad(12,9 \%)$ e namoro e casamento $(4,9 \%)$. data da entrevista, a média encontrada foi Tais resultados referem-se ao relato de, ao de 5 eventos por gestante, com o mínimo menos, um evento estressor presente em de 1 e máximo de $14(\mathrm{dp}=2,2)$ cada categoria (Tabela 1$)$.

Tabela 1 - Frequência e porcentagem de eventos estressores, referidos por categoria, entre gestantes de uma maternidade pública. Ribeirão Preto, 2010

\begin{tabular}{lcc}
\hline Categoria de eventos estressores & N & \% \\
\hline Saúde & 445 & 99,1 \\
Família e sociedade & 229 & 51,0 \\
Relações conjugais & 218 & 48,6 \\
Finanças & 189 & 42,1 \\
Trabalho & 154 & 34,3 \\
Migração & 147 & 32,7 \\
Reação de luto ou perda & 141 & 31,4 \\
Educação & 114 & 25,4 \\
Aspectos legais & 58 & 12,9 \\
Namoro e casamento & 22 & 4,9 \\
\hline
\end{tabular}


Ao analisar cada evento estressor isoladamente, $\mathrm{o}$ mais frequente foi “gravidez indesejada" (60,58\%), "gravidez desejada" (37,86\%), “dificuldade financeira média" (31,85\%) e "mudança para a mesma cidade" $(26,28 \%)$.

Dentre os eventos mais frequentes, aqueles que obtiveram maior impacto de estresse, ou seja, eventos que foram considerados geradores de estresse alto ou acentuado, destacam-se: "gravidez indesejada" (21,6\%), “dificuldades financeiras médias” (15,3\%), “brigas familiares" $(8,9 \%)$, "brigas com marido" $(8,9 \%)$ e "morte de familiares" $(8,7 \%)$.

Da amostra composta por 449 mulheres, 172 gestantes $(38,2 \%)$ foram encontradas como caso positivo pelo Questionário de Morbidade Psiquiátrica QMPA (escore $\geq 7$ ). Os casos positivos se referem às gestantes que apresentaram um ponto de corte igual ou maior a sete, no instrumento em questão, denotando problemas emocionais de relevada importância, com a probabilidade de serem portadores de doenças psiquiátricas. Esses dados foram obtidos a partir de estudo realizado com a mesma $\operatorname{amostra}^{(8)}$.

Verificou-se, neste estudo, a relação entre a ocorrência de eventos de vida na gestação e risco de morbidade psiquiátrica. Através do teste de KruskalWallis foi identificada maior ocorrência de eventos estressores entre gestantes positivas no Questionário de Morbidade Psiquiátrica (QMPA). O número total de eventos estressores apresentou correlação estatística positiva e significante em relação aos sintomas e queixas emocionais de gestantes $(p=0,001)$, ou 
seja, quanto maior o número de eventos estressores, maior o número de sintomas psiquiátricos encontrados.

Após análise de todas as categorias do instrumento IRLE separadamente, utilizando o mesmo teste não-paramétrico, os eventos das categorias educação $(p<0,05)$, finanças $(\mathrm{p}<0,001)$, problemas legais $(\mathrm{p}<0,05)$, migração $(p<0,05)$ e trabalho $(p<0,05)$ foram significantemente associados à morbidade psiquiátrica na gestação, como demonstrados na tabela a seguir (Tabela 2). A mesma associação para as categorias relacionamento, relações conjugais, família, perda ou luto e saúde não foram estatisticamente significantes.

Tabela 2 - Distribuição das gestantes $(\mathrm{n}=449)$, de acordo com as categorias de eventos estressores e casos no QMPA. Ribeirão Preto, 2010

\begin{tabular}{|c|c|c|c|c|c|c|}
\hline \multirow{3}{*}{ Categorias } & \multirow{3}{*}{$K$} & \multirow{3}{*}{$\mathbf{P}$} & \multicolumn{4}{|c|}{ Morbidade psiquiátrica } \\
\hline & & & \multicolumn{2}{|c|}{ Negativo } & \multicolumn{2}{|c|}{ Positivo } \\
\hline & & & Média & dp & Média & dp \\
\hline Relacionamento & 1,11 & ns & 0,06 & 0,23 & 0,04 & 0,23 \\
\hline Relações conjugais & 0,73 & ns & 0,69 & 0,89 & 0,79 & 0,99 \\
\hline Educação & 7,24 & $<0,05$ & 0,24 & 0,50 & 0,35 & 0,54 \\
\hline Família & 0,01 & ns & 0,64 & 0,73 & 0,65 & 0,80 \\
\hline Finanças & 18,44 & $<0,001$ & 0,36 & 0,52 & 0,56 & 0,51 \\
\hline Problemas legais & 8,42 & $<0,05$ & 0,09 & 0,29 & 0,20 & 0,43 \\
\hline Perda ou luto & 2,12 & $\mathrm{~ns}$ & 0,32 & 0,55 & 0,40 & 0,59 \\
\hline Migração & 7,44 & $<0,05$ & 0,28 & 0,45 & 0,41 & 0,50 \\
\hline Saúde & 1,42 & $\mathrm{~ns}$ & 1,33 & 0,55 & 1,26 & 0,53 \\
\hline Trabalho & 4,94 & $<0,05$ & 0,37 & 0,62 & 0,53 & 0,76 \\
\hline Número de eventos & 10,36 & 0,001 & 4,53 & 2,08 & 5,28 & 2,38 \\
\hline
\end{tabular}

Obs.: $K$-teste de Kruskal-Wallis (análise de variância não-paramétrica Ns-não significante; $p$-nível de significância estatística; dp-desvio padrão 
Identificou-se, ainda, através do teste estatístico do qui-quadrado, um ponto de corte referente à associação entre número total de eventos estressores e morbidade psiquiátrica. Encontrou-se que, das $171(38,1)$ gestantes positivas no QMPA, $72 \quad(16,1 \%)$ reportaram ter vivenciado mais de 5 eventos estressores, comparadas a $278(61,9 \%)$ gestantes negativas no QMPA, dessas, $81(18,0 \%)$ gestantes vivenciaram 5 ou mais eventos estressores. O grupo que reportou 5 ou mais eventos estressores, portanto, apresentou mais sintomas emocionais do que o grupo com menos eventos estressores, sendo tal resultado significante estatisticamente (chi-square, value $=7,3 ; \mathrm{p}<0,001)$.

\section{Discussão}

Ao analisar cada categoria do instrumento IRLE separadamente, houve correlação estatística positiva e significante em relação aos sintomas e queixas emocionais para as categorias educação $(p<0,05)$, finanças $(p<0,001)$, problemas legais $(p<0,05)$, migração $(\mathrm{p}<0,05)$ e trabalho, e, dessas, se destaca a categoria finanças, a qual foi fortemente relacionada à morbidade psiquiátrica em gestantes. Esse achado está consistente com outra pesquisa brasileira ${ }^{(20)}$, composta, predominantemente, por mulheres funcionárias de uma universidade pública do Rio de Janeiro, quando foi aplicado o questionário GHQ-

12 (General Health Questionnaire), utilizado para rastreamento de transtornos psiquiátricos menores (depressão e 
ansiedade) e uma entrevista para avaliação de eventos de vida produtores de estresse. O evento "ter passado por dificuldades financeiras graves" foi relatado com maior frequência e foi positivamente associado à depressão e ansiedade .

O fato de a maioria da amostra deste estudo apresentar baixa renda per capita, $(71,6 \%$ tinham renda de até cinco salários mínimos) poderia predispor essas mulheres a maiores situações adversas devido à instabilidade econômica. Os eventos estressores e traumáticos tenderiam ser mais frequentes entre grupos de menor status socioeconômico ${ }^{(21)}$, a pobreza também parece estar relacionada a problemas mentais, além de maior criminalidade e uso de substâncias ${ }^{(22)}$.

A literatura identifica que a mulher é potencialmente mais sujeita ao estresse do que o homem devido, principalmente, às profundas exigências e transformações nos papéis dessa na economia, na família e na sociedade, sendo que a própria gestação é considerada como importante fonte de estresse $^{(23-24)}$. Neste estudo, a maioria das gestantes referiram a gestação como evento estressor, sendo a "gravidez indesejada" $\mathrm{o}$ evento referido com impacto de estresse acentuado por 97 gestantes $(21,6 \%)$.

Encontrou-se correlação estatística positiva e significante entre a quantidade apresentada de eventos estressores e a morbidade psiquiátrica $(p<0,01)$. Esses dados indicam que o acúmulo de situações estressantes, no decorrer do ano, poderia causar desfechos negativos na saúde mental de gestantes em função do desgaste físico e psíquico envolvidos. Esses achados estão de acordo com outros 
estudos prévios conduzidos no Brasil e em outros países. Gestantes que vivenciam mais eventos estressores, durante o período gestacional, parece que apresentam mais sintomas depressivos e maior percepção de impacto do estresse $^{(25)}$. Outros estudos ${ }^{(26)}$ verificam que, além das associações positivas entre o número de eventos e danos à saúde mental, há também possível relação causal entre eventos estressores e o desenvolvimento de sintomas físicos e emocionais.

Pesquisas na literatura médica apresentam diversos prejuízos físicos relacionados ao maior número de eventos estressores. Neste estudo, foi identificada relação significativa entre a presença de 5 eventos no decorrer dos 12 meses anteriores à pesquisa e sintomas de distúrbio psiquiátrico. A exposição a cumulativos eventos (mais de 5) foi relacionada, portanto, com desfechos negativos na saúde mental de gestantes em função do desgaste envolvido.

\section{Conclusão}

Os resultados deste estudo revelaram a relação de morbidade psiquiátrica e a presença de cinco ou mais eventos estressores. Esses dados apontam para a importância da avaliação de eventos estressores durante a gestação, como uma das formas de prevenção e tratamento de possíveis transtornos psiquiátricos. Conforme dados da literatura, os eventos estressores podem prejudicar a saúde mental da gestante bem como o desenvolvimento do bebê. Porém, a realização de estudos longitudinais com gestantes, para investigar as consequências dos eventos 
estressores, ao longo da vida dessas, buscando verificar a associação de eventos estressores e o aparecimento de problemas psiquiátricos e outras complicações, dará mais consistência a esses dados. A identificação dos fatores de risco e protetores também poderia auxiliar no planejamento de intervenções com gestantes que estão passando por situações estressantes.

Conclui-se que os resultados deste estudo apresentam contribuição clínica na medida em que se considera importante a avaliação dos eventos estressores vivenciados durante o período gestacional. Tal avaliação, realizada pelos profissionais de saúde, pode contribuir para o tratamento, educação e promoção da saúde pública, ao ajudar as mulheres a reforçar os seus estilos de enfrentamento, levando-as a melhor gestão do autocuidado.
Referências

1. Paykel ES. The interview for recent life events. Psychol Med. 1997;27:301-10.

2. Chrousos GP. Stress and disorders of the stress system. Nat Rev Endocrinol. 2009;5: 374-81.

3. Margis R, Picon P, Cosner AP.
Relação entre estressores, estresse e ansiedade. Rev Psiquiatr Rio Gd Sul. 2003;25 supl 1:65-74.

4. Muhwezi WW, Agren H, Neema S. Life events associated with major depression in Ugandan primary healthcare patients: issues of cultural specificity. Int $\mathrm{J}$ Soc Psychiatry. 2009;54(2):144-63. 
5. Whiteheard N. The Sistema Único de Saúde. Rev Saúde

Relationship Between Individual Life

Events and Preterm Delivery. RTI Press

publication No. RR-0003-0809. Research

Triangle Park, NC: RTI International.

Disponível em:http://www.rti.org/rtipress, 2008 Sept.

6. Mulder EJH. Prenatal maternal

stress : effects on pregnancy and the (unborn) child. Early Human Develop. 2002 June;70:3-14.

7. Faslow SM, Robinson E, Whitehead A. Violence during pregnancy: associations with pregnancy intendedness, pregnancy-related care, and alcohol and tobacco use among a representative sample of New Zealand women. Aust N Z J Obstet Gynaecol. 2008;48(4):398-404.

8. Pinheiro S N, Laprega MR, Furtado EF. Morbidade psiquiátrica e uso de álcool em gestantes usuárias do
Pública. 2005;39(4):593-8.

9. Spietz A, Kelly J. The importance of maternal mental health during pregnancy: theory, practice, and intervention. Public Health Nurs. 2002 May-Jun;19:153-5.

10. Hansen D, Jou HC, Olsen J. Serious life events and congenital malformations: a national study with complete follow-up. Lancet. 2001 Jun;30:357.

11. Rice F, Thapar A. The impact of gestacional stress and prenatal growth on emotional problems in offspring:a review. Acta Psychiatr Scand.a 2007 March; 115:171-83.

12. Egliston K-A, Catherine M, Marie-Paule A. Stress in pregnancy and infant HPA axis function: Conceptual and methodological issues relating to the use of salivary cortisol as an outcome 
measure. Psychoneuroendocrinology. 2007 Jan;32(1):1-13.

\section{Glover V; O’Connor TG.}

Effects of antenatal stress and anxiety. $\mathrm{Br}$

J Psychiatry. 2002;180:389-91.

14. Maldonado MTP. Psicologia da Gravidez. Petrópolis: Vozes; 1997.

15. Coutinho DS, Baptista MN, Morais PR. Depressão pós-parto: prevalência e correlação com o suporte social. Infanto-Rev Neuropsiq da Inf. e Adolesc. 2006; 10(2):63-71.

16. Santana W. Estudo epidemiológico das doenças mentais em bairro de Salvador. Salvador: Instituto de Saúde da Bahia; 1982 (Série de Estudos em Saúde).

17. Paykel ES. The evolution of life events research in psychiatry. J Affect Disorders. 2001;62:141-9.

18. Paykel ES, Cooper Z, Ramana

RH. Life events, social support and marital relationships in the outcome of severe depression. Psychol Med. 1996;14:559-68.

19. Pardoen D, Bauwens F, Tracy A, Martin FM. Self-esteem in recovered bipolar and unipolar outpatients. $\mathrm{Br} \mathrm{J}$ Psychiat. 1993;163:755-62.

20. Zimmerman-Tansella C, Donini S, Galvan U, Rizzetto A. Social support, adversities and emotional distress in an Italian community sample. $\mathbf{J}$ Clinical Epidemiol. 1993;46: 65-75.

21. Marks MN, Wieck A, Checkley SA, Kumar R. Contribution of psychological and social factors to psychotic and non-psychotic relapse after childbirth in women with previous histories of affective disorder. J Affect Disorders. 1992;29:253-64.

22. Lopes CS, Faerstein E, Chor D. Eventos de vida produtores de estresse e transtornos mentais comuns: resultados 
do estudo pró-saúde. Cad Saúde Pública. nov-dez 2003;19(6):1713-20.

23. Turner RJ, Avison WR. Status Variations in $\quad$ Stress exposure:Implications for the Interpretation of Research on Race, Socioeconomic Status and Gender. J Health Soc Behav. 2003 Dec;44(4):488505.

24. Rossi AM. Estressado eu ?. Porto Alegre: RBS Publicações; 2004.
25. Muhwezi WW,Agren H,Neema S. Life events associated with major depression in Ugandan primary healthcare patients: issues of cultural specificity. J Soc Psychiatry. 2008 Mar;54(2):144-63.

26. Cooke DJ. The significance of life events as a cause of psychological and physical disorders. In: Cooper B. Psychiatric Epidemiology. London: Croom Helm; 1987. p. 67-80.

\section{Como citar este artigo:}

Esper LH, Furtado LH. Associação de eventos estressores e morbidade psiquiátrica em gestantes. SMAD, Rev. Eletrônica Saúde Mental Álcool Drog. (Ed. port.) [Internet]. 2010 [acesso: dia mês abreviado com ponto ano]; 6(Especial):368-86. Disponível em: Endereço Eletrônico Visitado. 